\title{
Éditorial
}

\section{Notre revue fête ses 40 ans}

En septembre 1966, la Société française de radioprotection publiait le premier numéro de son journal Radioprotection. Sans pour autant tomber dans la nostalgie, cette année est particulièrement riche en anniversaire avec les 20 ans de Tchernobyl, les 50 ans de l'UNSCEAR, et un peu plus jeune que l'UNSCEAR, les 40 ans de Radioprotection.

En cette occasion nous avons voulu changer de couverture ; c'est l'évènement le plus directement visible, tout en gardant nos couleurs fétiches : le vert et le fuschia. Le trèfle radioactif très stylisé (un peu trop au goût de certains), a disparu. Il fait place à un trèfle très visible, quelques taches fuschia, pour égayer un peu ce symbole de la radioactivité.

Nous avons voulu également vous rappeler notre premier numéro en reproduisant sa table des matières, son premier éditorial, écrit par Louis Bugnard (voir pp. 273-274), et nous n'avons pas échappé à la tentation de brosser la vie de notre journal durant ces 40 ans.

Que dire du contenu du premier numéro de 1966 ? Un commentaire sur les accidents, déjà les problèmes de mesure des neutrons, une méthode analytique pointue, les activités de radioprotection d'un laboratoire universitaire, un article de dosimétrie biologique et un article sur les effets hématologiques. Déjà on percevait les difficultés qu'allait vivre cette revue, et ses richesses liées à sa grande diversité. Pourtant, malgré des articles et un lectorat très diversifiés, parfois difficiles à gérer, nous sommes toujours là quarante ans après et, j'en suis sûr, ce n'est pas fini.

L'éditorial de Louis Bugnard reste d'actualité, la revue qu'il appelait de tous ses vœux pour «donner aux membres de la société la possibilité d'exposer et de confronter les résultats les plus récents obtenus dans les domaines si divers de la radioprotection » a rempli tout son rôle. Elle s'est largement ouverte au-delà des membres de la SFRP, c'est là une de ses réussites. Le numéro que vous avez sous vos yeux en est une nouvelle illustration.

Autre point fort de ces $\mathbf{4 0}$ ans de publication, la présence dès le début, de notre ami Daniel Blanc, membre de la première commission des publications présidée alors par Henri François. Il est toujours présent, et bien présent au sein de notre comité, c'est lui qui m'a attiré dans cette aventure et il était tout naturel que je lui demande de nous brosser en quelques lignes la vie de notre revue ; encore merci Daniel. Que beaucoup l'imitent et notre revue perdurera encore de très nombreuses années.

DOI: $10.1051 /$ radiopro:2006014

RADIOPROTECTION - VOL. 41 - Nº 3 (2006) 
ÉDITORIAL

Aujourd'hui, notre revue est entrée dans l'ère moderne. Nos articles sont consultables sur Internet, sa vie et sa fabrication se font par voie électronique, nos tirés-à-part ont disparu, ce sont des fichiers PDF. Bref, un système de gestion plus moderne qui, de plus, satisfait notre trésorier, car je peux vous l'affirmer, jamais un trésorier ne nous a oublié.

Depuis 1966, plus de 600 articles ont enrichi nos colonnes, et il faut que cela dure. Je sais que par nos métiers, très divers, de la radioprotection, tous nos collègues n'ont pas spontanément la volonté ou la possibilité d'écrire. Pourtant il le faut, en français pour nos collègues, en anglais lorsque l'on veut défendre sur le plan international, l'un de nos résultats ou l'une de nos positions. Le comité fait des efforts constants pour « rameuter les troupes », nous allons aussi solliciter les sections, forces vives de notre association. C'est par vos articles que l'on pourra espérer fêter un second quarantième anniversaire.

Enfin, cette revue n'a pu durer que parce qu'elle a eu le soutien des différents présidents de notre société, et c'est toujours la réalité. Aujourd'hui plus encore, le mot de notre président André Aurengo est là pour en témoigner.

La revue a connu plusieurs éditeurs, mais je n'en ai connu qu'un, EDP Sciences. Là encore la SFRP a fait un bon choix, elle ne peut que s'en féliciter.

Pour remercier et honorer de la meilleure façon tous les auteurs qui ont fait le succès de notre journal ces quarante dernières années, n'hésitez pas à nous envoyer des articles.

Une fois de plus, tous à vos plumes et pour 40 ans encore, au moins.

Henri Métivier

Président du comité de la revue 